\title{
La parenté « nouvelle » ou le retour de la substance
}

\section{Emmanuel Désveaux}

\section{OpenEdition}

\section{Journals}

Édition électronique

URL : https://journals.openedition.org/jsa/10809

DOI : 10.4000/jsa. 10809

ISSN : 1957-7842

\section{Éditeur}

Société des américanistes

\section{Édition imprimée}

Date de publication : 23 juillet 2009

Pagination : 213-223

ISSN : 0037-9174

\section{Référence électronique}

Emmanuel Désveaux, «La parenté « nouvelle » ou le retour de la substance », Journal de la Société des américanistes [En ligne], 95-1 | 2009, mis en ligne le 22 février 2014, consulté le 04 septembre 2022. URL : http://journals.openedition.org/jsa/10809; DOI : https://doi.org/10.4000/jsa.10809

Ce document a été généré automatiquement le 4 septembre 2022

Tous droits réservés 


\title{
La parenté « nouvelle » ou le retour de la substance
}

\author{
Emmanuel Désveaux
}

«Er will hier alte Freundschaft und Verwandtschaft wiedersehen

- Hat er denn hier Verwandtschaft?

- Ja und nein, wie man's nehmen will. "1

Theodore Fontane, Effi Briest

1 En publiant La parenté, Laurent Barry se propose d'apporter sa pierre à cet édifice qui se situe au cœur même de l'anthropologie classique, à savoir le champ de la parenté ${ }^{2}$. Il nous offre pour ce faire un fort volume de plus de huit cent pages, à la base documentaire étendue et à l'argumentation dense, et qu'une écriture directe et sans fioriture s'attache à rendre le plus accessible possible. Le texte principal s'enrichit, outre de la bibliographie, d'un glossaire, ce qui traduit ce même souci d'accessibilité ${ }^{3}$. La collection non spécialisée d'un grand éditeur parisien qui l'accueille atteste que ces efforts de lisibilité ont été appréciés et on ne peut que s'en réjouir. Mais ce livre n'est pas pour autant un manuel d'introduction à la parenté ; il défend une thèse qui se veut nouvelle et souhaite installer un nouveau paradigme. Quant au titre, La parenté - rien de plus, mais surtout rien de moins -, il peut paraître soit comme le signe d'une grande économie dans l'expression, soit comme celui d'une très grande ambition dans le propos.

2 Le livre débute assez naturellement par une généalogie critique du domaine (chapitres I et II). Si les points abordés sont multiples, il en ressort surtout que la théorie lévistraussienne de l'échange matrimonial, dans sa vocation universelle du moins, est désormais dépassée. C'est dans le chapitre III, intitulé « Une théorie des groupes de parenté », que se concentre l'argument central du livre: une déclinaison et une combinatoire, alors exposées sur un plan strictement abstrait, de ce qui semble à l'auteur constituer les principes fondamentaux de la parenté. La suite du livre se présente comme une série d'essais, richement fournis en données ethnographiques, riches également en démonstrations partielles, parfois fort suggestives, qui viennent à l'appui des différentes 
« sortes » de parenté qui découlent du chapitre III, ce noyau canonique, si l'on peut dire, du travail de Barry.

3 Revenons donc à ce chapitre et examinons scrupuleusement sa progression. D'emblée, Barry énonce un double postulat: la parenté existe dans toutes les sociétés humaines. Elle procèderait partout d'un même sentiment d'identité partagée, équivalent à un rejet de l'inceste (mais d'où vient l'inceste ? le grand mérite de la théorie de Lévi-Strauss était d'avoir répondu à la question ou, tout du moins, de l'avoir neutralisée en affirmant l'universalité de sa prohibition). Cette identité partagée commande des groupes, dits "groupes de parenté». Les groupes en question sont en réalité des groupes de génération, ce qui revient à dire qu'on y appartient par sa naissance. Ils sont susceptibles d'être formés selon quatre modes distincts, qui correspondent à autant de conceptions de la transmission de l'identité. Celle-ci peut être soit utérine (on tient uniquement de sa mère), soit agnatique (on ne tient que de son père), soit parallèle (ici, les garçons tiennent de leur père et les filles de leur mère), soit indifférenciée (chaque sexe tient de façon indistincte des deux sexes). Précisons que, pour l'auteur de La parenté, cette transmission se reproduit semblable à elle-même à chaque génération et que se créent ainsi des lignées, hormis dans le cas de l'indifférenciation, bien entendu, qui par définition entrave l'émergence d'une telle linéarité.

Barry voit dans la capacité de détecter des ressemblances physiques entre une progéniture et ses géniteurs le fondement « cognitif» de cette perception d'une identité commune aux uns et aux autres. On doit sérieusement mettre en doute la valeur de cette observation, soutènement pourtant de toute la démonstration. Si l'art d'établir des ressemblances entre individus apparentés est bien une grande caractéristique de nos cultures occidentales, son universalité nous semble douteuse. Nous ne l'avons pas rencontrée sur nos terrains nord-américains et elle est virtuellement absente de la littérature ethnographique américaniste. Certes, on peut admettre que les Amérindiens aient perdu, au cours de leur longue évolution parallèle à celle du reste de l'humanité, cette faculté, voire y aient renoncé en fonction des contraintes que leur imposaient leurs cultures, mais l'argument ne vaut plus dès lors que l'on tente d'imaginer les phases cruciales du passage de la nature à la culture. Car la contradiction surgit immédiatement ici : d'une part, nos lointains ancêtres, candidats à une hominisation achevée et donc inventeurs de la parenté (nous resterons personnellement lévi-straussien sur ce point), fondent une systématique de la classification et de l'identification des individus au sein de groupes sur les seules vertus de la ressemblance; d'autre part, ils renoncent à l'essentiel des avantages que procure ce dispositif en créant les «filières" purement utérines ou purement agnatiques ou, encore, parallèles, alors même que les phénomènes de ce type ne respectent pas la division entre les sexes, ni même l'unilinéarité. Nos propres réunions familiales en attestent parfaitement: indépendamment de son caractère hautement subjectif, la ressemblance entre parents emprunte toute sorte de voies, et notamment celle de la collatéralité. C'est parfois la fille qui ressemble au père ou à l'oncle ; le petit-fils à la grand-mère ou à la tante, etc.; ou encore ce sont des cousins qui partagent un air de famille et, ce, indépendamment bien sûr de leur caractère parallèle ou croisé.

5 Barry avance ensuite la notion assez étrange « de parcours cognitif » qui lui permet de hiérarchiser les modes de constitution des groupes de parenté selon un gradient de plus ou moins grande adhérence à la sensibilité immédiate (pour reprendre ici les termes de Lévi-Strauss). Il lui faut du coup préciser qu'après le critère de ressemblance, ce qui 
importe dans l'élaboration de ce sentiment de parenté, c'est l'évidence de la maternité, autrement dit l'ensemble du processus qui préside à la procréation humaine à partir de la conception et qui comprend la gestation, l'accouchement et l'allaitement. En combinant l'impératif cognitif de la ressemblance avec celui qu'offre la prégnance de la maternité, on obtient donc la déclinaison suivante : mode indifférencié (on ressemble à qui que ce soit des deux côtés), mode parallèle (les garçons ressemblent aux hommes, les filles aux femmes), mode utérin (c'est ici seulement qu'intervient la prééminence du facteur féminin dans le processus de procréation), mode agnatique enfin, supposé être le plus éloigné de la réalité perçue. Cette hiérarchie possède des relents d'évolutionnisme, puisque le mode indifférencié apparaît comme le plus performant d'un point de vue cognitif, mesuré évidemment à l'aune de nos conceptions contemporaines en matière de reproduction biologique, et s'instaure du coup comme référence. Or c'est également comme par hasard - celui de l'Occident. Reconnaissons toutefois que l'auteur de La parenté esquive l'écueil évolutionniste avec subtilité puisque la hiérarchie qu'il propose se reflète uniquement dans la distribution statistique des systèmes à la surface du globe et à l'aune de l'histoire humaine dans sa totalité (mesurée sur quelles bases et appréciée selon quelles données ?). L'indifférenciation aurait été plus courante que le parallèle, puis viendrait, en terme de fréquence, l'utérin et ensuite l'agnatique qui se révèlerait en fait très rare. Il nous faut ici anticiper sur les chapitres suivants et mentionner quelques corrélations ethnographiques pour mieux suivre la classification de Barry. En gros, "l'indifférencié » c'est, ainsi que nous l'avons déjà signalé, nous, les Occidentaux, et quelques autres. Les " parallèles » correspondent aux systèmes de parenté fondés sur la distinction entre cousins parallèles et cousins croisés et recouvrent globalement les structures élémentaires de la terminologie, devenue classique, de Lévi-Strauss. Les utérins renvoient aux sociétés qui s'adonnent au mariage dit " arabe ». Ici, Barry s'appuie sur ses propres travaux théoriques antérieurs ancrés dans l'ethnographie de plusieurs populations peules ${ }^{4}$. Outre toute la littérature sur les Arabes justement, il mobilise l'exemple de Madagascar pour illustrer cette forme de parenté qui privilégie le mariage avec la cousine parallèle patrilatérale. La parenté utérine doit uniquement se comprendre, dans le cadre de l'endogamie (mais l'option en tant que telle reste à expliquer), comme le résultat d'une stratégie tendant à repousser le plus longtemps possible le contact avec la lignée utérine qui avait, à la génération précédente, engendré la mère. Les agnatiques, enfin, seraient incarnés par les seuls Han de Chine. On entre alors dans l'ordre de la rareté absolue à l'échelle de l'évolution et de l'histoire humaines. En bref, l'argument sollicite les faits majoritaires en renfort du sens commun, lequel, on l'a $\mathrm{vu}$, revient au régime indifférencié. Pour ce qui est des très rares exemples d'" agnatisme " intégral, on se demande si leur existence ne tient pas à la nécessité d'illustrer une des possibilités logiques de la matrice conceptuelle de Barry. Nul doute, en tout cas, que cette déclinaison de premier niveau ne bouscule quelque peu les catégories conceptuelles auxquelles sont accoutumés les spécialistes du domaine. On se demandera toutefois si tous les efforts de redéfinition et de réorganisation du champ, auxquels nous assistons ici, ne procèdent pas en grande partie du fait que le mariage arabe - reliquat de la théorie lévi-straussienne de l'échange matrimonial - a représenté, dans la réflexion antérieure de Barry, un aspect central. À travers la notion de " parenté utérine ", un des quatre piliers sur lesquels repose le premier niveau du système général proposé, ce type de mariage retrouve un rang et une dignité qui lui étaient jusqu'à présent déniés... Somme toute, ce n'est pas là que réside l'étonnement majeur que nous réserve la lecture du chapitre III de La parenté. 
6 Barry suppose ensuite que le phénomène de transmission de qualités intrinsèquement sexuées (ce que nous venons de voir) ne s'opère pas seulement sur un plan intergénérationnel, mais également lors de la relation sexuelle. Précisons qu'il s'agit de la relation sexuelle répétée et plus ou moins stabilisée, autrement dit le mariage. Et nous nous retrouvons alors devant une nouvelle déclinaison qui duplique la précédente (principe cognatique, principe parallèle, principe utérin, principe agnatique). Ainsi, par le mariage, l'individu, tantôt masculin, tantôt féminin, transmet partiellement ou entièrement des qualités du ressort de son propre sexe à son conjoint. Nous reviendrons plus loin sur ce qu'implique cette conception de la transmission horizontale (et non plus seulement verticale). Remarquons simplement, pour l'instant, qu'elle ne saurait être équivalente au premier niveau dans la mesure où nous entrons dans un registre de relations sociales qui s'avère en règle générale moins stable, propre à introduire de la confusion, à cause des divorces ou des veuvages, d'une part, de la multiplicité éventuelle des unions par polygamie, d'autre part. Toutefois, Barry ne s'embarrasse plus guère de ce genre de difficulté, parvenu à ce stade de sa démonstration, puisqu'il se contente en l'occurrence de décrire ce passage de la verticalité à l'horizontalité, à l'intérieur même de sa propre construction argumentative, comme s'il s'agissait d'une simple « extension du groupe de parenté dans l'affinité ». Il y a là, de notre point de vue du moins, une erreur dans la méthode suivie.

Car l'expression « l'extension dans » suppose que l'affinité soit déjà prédéfinie. Or, s'il en est ainsi, cela signifie que les progrès sont nuls par rapport à Morgan qui intitulait son livre Systems of consanguinity and affinity in the human family. Et comment pourrait-elle être définie cette affinité, en l'absence de toute autre indication, sinon en pendant ou, plutôt, en miroir de la consanguinité? Du coup, on reste confondu devant tant de candeur de la part d'un auteur qui, souhaitant révolutionner le champ, revient à un type de formulation dont de très nombreux développements de la théorie de la parenté, de Kroeber à Lévi-Strauss (pour une fois, ils se situent du même côté), cherchaient justement à s'affranchir. Schneider est probablement l'auteur qui est allé le plus loin dans cette déconstruction de la dualité consanguinité/affinité et du primat du premier sur le second ${ }^{6}$. Tandis que, pour sa part, quelqu'un comme Eduardo Viveiros de Castro propose de considérer que l'affinité est première et triviale dans le cas des Amérindiens d'Amazonie. De sorte que c'est la consanguinité qui s'avère source de tensions sociologiques, devant s'élaborer à partir d'un extérieur sociologique qu'incarne, de façon médiane il est vrai, l'affinité7.

Quoi qu'il en soit, logique avec lui-même, lui qui n'y voit donc qu'une simple extension, Barry décalque les régimes de transmission d'identité par mariage sur ceux qu'il a édictés pour la génération, son objectif étant de dresser une table des permutations combinant les deux régimes, table censée rendre compte de tous les systèmes de parenté. La tâche lui sera d'autant plus aisée qu'il envisage toutes les demi-mesures ad hoc lui permettant ici ou là d'ajuster son modèle à la réalité ethnographique. Mais convenons qu'il n'est pas le premier anthropologue à procéder ainsi ! Les doutes surgissent surtout à cause du contenu sexué de l'« incorporation d'identité » qu'il voit à l'œuvre à travers le mariage. On peut admettre que, dans certaines cultures, le mariage transforme l'épouse en une sorte d'auxiliaire absolu des attributs sociologiques de son mari (ou l'inverse, dans d'autres sociétés, bien que cela soit plus rare). En revanche, on ne voit pas ce à quoi correspond l'opération au titre du régime dit " parallèle » (qui correspond à celui des cousins croisés et de l'échange restreint selon la doctrine lévi-straussienne classique). 
Nous citons : « chacun des conjoints transmet à l'autre un principe propre à son sexe et éventuellement, secondairement, celui propre à l'autre sexe» (p. 203). Esquissons une traduction dans un autre langage : si je me trouvais être un dravidien, je deviendrais par le mariage une dravidienne et ma nouvelle épouse un dravidien, tout en récupérant, peut-être en vertu d'un processus d'échange-transmission à double sens - à l'origine parfaitement obscure -, un peu de caractère dravidien et en restituant en même temps à mon épouse un peu de sa nature dravidienne. Autant dire, à cet endroit, que Barry nous plonge dans la plus grande des perplexités...

Le dernier régime, l'indifférencié, s'avère de prime abord nettement moins difficile à entendre. De fait, il traduit parfaitement un dispositif culturel qui, pour être fort éloigné du sens commun, nous est cependant profondément familier dans la mesure où il s'agit de celui qu'a imposé le christianisme à l'occident depuis presque deux mille ans. On sait en effet que una caro, " une seule chaire», constitue le fondement de la doctrine de l'Église en matière de mariage. En vertu de ce dogme, la rencontre charnelle de l'homme et de la femme trouve sa légitimité dans la fusion de leurs corps respectifs en une unité indissociable. Pour l'Église, le mariage est avant tout un sacrement, miroir de l'union du corps que constitue l'ensemble des croyants et de Dieu. On sait que la doctrine a impliqué une exogamie maximale, afin que la fusion soit pleinement signifiante. Il faut signaler ici que, sur ce point, Barry, dans la foulée d'auteurs tels que Jack Goody ${ }^{8}$, se montre assez conséquent : il affirme la nécessité d'intégrer l'Occident - et son histoire dans une théorie générale de la parenté et de ne pas se contenter de l'étude des sociétés exotiques. Une grande place est donc accordée dans l'ouvrage à la restitution des travaux d'une nouvelle génération d'antiquisants, tel en particulier Jérôme Wilgaux ${ }^{9}$ sur la Grèce dont les travaux vont dans le même sens que ceux de Barry sur le mariage arabe, à savoir dans le sens d'un évitement de la lignée utérine. Mais, là encore, en anticipant sur des développements qui interviennent plus loin dans le livre, il nous semble que ce recours à l'histoire a surtout le mérite pour Barry de faire un pont entre certains mondes exotiques et le monde occidental, entre la parenté utérine et l'una caro. Pont qui, selon nous, serait celui qui permet également de relier les tendances respectives à l'endogamie et à l'exogamie. Au-delà de cet aspect, le recentrement opéré par Barry sur l'histoire occidentale nous aide à mieux dégager ce qui constitue l'argument caché de tout le livre.

Nous avons affaire, tout banalement, à la notion de substance, laquelle se transmet de génération en génération à travers le « sang ", le lait maternel - Barry soutient, contre Françoise Héritier, que celui-ci commande dans le monde arabe des interdits matrimoniaux en son nom propre et non comme substitut du sperme--, ou encore les diverses émissions ou sécrétions génitales. Il convient bien, dès lors, de parler de substance, car on ne voit pas ce qui aurait non seulement la capacité de se transmettre d'une génération à l'autre et de transmettre en même temps des attributs physiques, voire moraux, tout en étant susceptible d'affecter l'identité de l'autre, par le biais de la relation sexuelle, c'est-à-dire de le contaminer. Reconnaissons alors qu'il fallait une bonne dose de courage - ou de naïveté - à Barry pour s'être engagé de façon si radicale dans la construction d'un paradigme aussi hasardeux. Et ce, alors même que ce n'était peut-être pas tant le sien que celui d'Héritier. L'auteur de La parenté se montre ici un disciple dévoué, le différend sur le sperme, évoqué un peu plus haut, s'avérant au fond assez mineur. Il se montre aussi fidèle disciple en poursuivant la tâche du démantèlement de l'échange matrimonial lévi-straussien, qu'elle avait esquissé, d'abord de manière assez timide, dans L'exercice de la parentée ${ }^{10}$, puis de façon plus ouverte, mais aussi assez erratique, dans ses textes suivants par le biais du concept, hautement bancal, 
d'inceste du deuxième type $\mathrm{e}^{11}$. On a l'impression que Barry entend aujourd'hui reprendre le flambeau d'une heuristique de la parenté dérivée de la substance, tout en cherchant à remédier aux faiblesses intrinsèques des travaux d'Héritier, au demeurant reçus pendant des années dans un silence poli par la communauté des anthropologues ${ }^{12}$. D'où les efforts de formulation et de systématisation, dont témoigne le chapitre III de La parenté.

11 Cela étant, si une théorie de la parenté qui favorise une détermination de par le "sang " et par la contamination semble gagner en rigueur formelle grâce à Barry, elle n'en reste pas moins profondément ethnocentrique. Elle correspond trop à l'idéologie occidentale pour ne pas susciter le doute quant à son universalité, qu'il s'agisse de l'idéologie traditionnelle d'una caro ou de celle, très contemporaine, du code génétique d'une part, de la suspicion généralisée d'inceste d'autre part (nous entendons par là la place centrale qu'a prise en Occident la thématique de l'inceste dans les relations familiales ordinaires sous l'influence de la pensée freudienne). L'inventeur de la théorie de la parenté, Morgan, pensait, il y a plus d'un siècle, que nous sommes, nous les Occidentaux, ceux dont les comportements et les interdits en matière de relations à nos proches se situent au plus près de la rationalité des origines, donc d'une certaine loi naturelle. Et bien entendu, pour lui, la supériorité absolue des manières occidentales - monogamie absolue (même pas de remariage après veuvage) et nomenclature dite " descriptive » - résidait dans le fait qu'elles seules permettaient de refléter dans l'état de culture, grâce au modèle de la famille nucléaire, ce que sont les vrais degrés de consanguinité - entre individus, lesquels relèvent évidemment de l'état de nature. Nul doute que l'inventeur de la théorie de la parenté participait au grand partage lorsqu'il rejetait les nomenclatures de parenté de la majorité des populations non occidentales dans la catégorie classificatoire à l'opposé des nôtres qui seraient descriptives. Il faisait des cultures de simples illustrations des errements de l'espèce humaine avant qu'elle ne parvienne enfin à découvrir les vertus du mariage puritain ${ }^{13}$. On serait tenté de dire que Barry procède à l'inverse de Morgan : il tend à reconnaître les linéaments de la parenté occidentale partout.

Pour terminer, il nous faut aborder deux questions qui restent pour nous comme des points aveugles de La parenté.

Premièrement, pourquoi Barry avance-t-il de façon masquée, n'osant pas nommer explicitement ce qui sert de fondement et de fil conducteur à son travail, à savoir cette fameuse substance ? L'image un peu surannée et fort ambiguë dont est parée la notion au sein de notre communauté scientifique explique probablement ce curieux non-dit. Elle éveille des soupçons à cause de sa connotation biologisante, mais aussi à cause des relents d'ethnocentrisme, palpables dans l'instant par n'importe quel ethnologue. Somme toute, la substance aujourd'hui ne peut se comparer avec la réciprocité qui s'était imposée à Lévi-Strauss, dans le sillage de Mauss, comme clé d'interprétation universelle des conduites humaines et qui était vouée à un grand rayonnement dans le climat un peu particulier de ressaisissement moral qui marqua l'après-guerre. Il est à craindre qu'en comparaison La parenté ne tombe un peu à plat. D'autant plus que Barry c'est tout à son honneur - ne souhaite manifestement pas s'engager sur la voie d'une détermination biologique de la parenté, refusant de céder aux sirènes d'une néonaturalisation qui résonne de façon de plus en plus stridente à la porte de notre discipline. Mais, du coup, tout son argument apparaît comme un peu déconnecté, hors du temps. Barry semble en être conscient au demeurant. Dans sa conclusion, il joue ainsi la modestie et va jusqu'à dénier à sa proposition théorique toute valeur générale ou toute dimension philosophique au-delà du strict domaine de la parenté, réduisant 
volontairement celui-ci à n'être en apparence que le sujet de discussions purement techniques.

Deuxième question: pourquoi l'Amérique, soit une large part de l'humanité, est-elle totalement absente d'une proposition théorique qui se présente d'emblée comme ayant valeur universelle? Le déni est d'autant plus choquant, notamment pour les américanistes, que l'auteur de La parenté ne prend jamais la peine de fournir la moindre explication pour justifier un tel « oubli ", serait-elle seulement d'ordre rhétorique. Son silence est si assourdissant que l'on se demande s'il ne relève pas d'un véritable acte manqué... Un tel manquement s'explique, me semble-t-il, par deux motifs qui s'emboîtent. Le premier tient au paysage intellectuel actuel dans notre discipline. Il se trouve en effet que, la partie nord du Nouveau Monde - qui fut pourtant avec Morgan, puis avec Kroeber, le foyer historique de la discussion - n'intéressant plus personne, les études américanistes de la parenté se sont focalisées pour l'essentiel depuis quelques décennies sur l'Amazonie. Dans ce cadre, une brillante école franco-brésilienne s'est constituée sous la houlette de Viveiros de Castro, composée en particulier d'Anne Christine Taylor, de Carlos Fausto, d'Aparecida Vilaça ou encore d'Isabelle Daillant ${ }^{14}$. Fidèles aux enseignements des Structures élémentaires de la parenté, les membres de ce groupe font de la réciprocité la clé d'interprétation privilégiée des comportements sociaux, en général, et matrimoniaux, en particulier. La teneur dominante des nomenclatures de parenté, décrites comme dravidiennes, les conforte dans cette interprétation. De plus, aucun d'entre eux ne renonce à la valeur universelle de leur paradigme fétiche. Dès lors, l'Amérique a pu sembler à Barry comme le "bastion », la «forteresse imprenable» de l'échange matrimonial, et il aurait eu toutes les bonnes raisons de renoncer à l'investir. Ajoutons une motivation plus technique. Dans sa réorganisation du domaine, s'il reclasse les systèmes reposant sur le mariage entre cousins croisés, associés généralement aux fameuses terminologies dravidiennes, comme "parenté parallèle», donc selon un principe de dévolution de l'«identité » qui appartient en propre à chaque sexe, il a cependant la tentation d'en préserver partiellement un fondement échangiste d'obédience lévi-straussienne. Du coup, ainsi que nous l'avons souligné plus haut, il est clair que la catégorie représente l'un des maillons parmi les plus faibles de son dispositif général. Autant éviter, dans ces conditions, de se jeter dans la gueule du loup !

Un second motif, plus profond, explique peut-être cette désertion face au Nouveau monde. Si le mot "substance" fait effectivement sens pour décrire des réalités ethnographiques amérindiennes, il ne s'agit pas du tout, en effet, de la même chose que celle qui sous-tend la vision de la parenté chez Barry. La substance amérindienne, comprise essentiellement là encore d'après l'ethnographie amazonienne où elle joue un rôle majeur, est cette consistance charnelle qui leste les êtres vivants en les rendant sujets à une prédation généralisée dont l'anthropophagie, réelle ou parfois seulement imaginaire, demeure la figure obligée. C'est même, pourrait-on dire, cette saturation de matière que véhiculent les entités sociales - à l'instar des différentes espèces animales -, par l'intermédiaire de chacun de leurs membres, et qui est susceptible d'entrer en bloc dans un commerce avec autrui, dont les mécanismes de la parenté seraient l'expression privilégiée ${ }^{15}$. Nous sommes fort éloignés de la fluidité, idéalement divisible, à la fois contaminante et soluble, vecteur de la potentialité variable de vertus ou de tares d'une génération à l'autre en raison de ce que nous appelons couramment l'hérédité, voire d'un individu à l'autre dans la relation sexuelle, qui caractérise la "substance " à l'Occidentale et qui, comme nous le croyons, campe en arrière-fond de l'imaginaire 
théorique de Barry. L'incompatibilité est totale. L'Amérique ne peut donc qu'être exclue de l'ordre de La parenté dans le sens où il l'entend...

Oserait-on écrire que, à notre avis, si elle était pleinement assumée - ce qui n'est pas le cas, car Barry, au fond, à l'instar de la plupart de nos collègues, ne veut en aucun cas renoncer à l'universalité -, cette exclusion ne nous gênerait pas outre mesure ? Car elle reflète en creux, par inadvertance presque, ce qu'est, selon nous, la réalité de la parenté en Amérique, à savoir une catégorie de l'entendement amérindien qui réfute l'hérédité biologique, tout en affirmant clairement, à la différence de certaines cultures océaniennes, que la relation hétérosexuelle est la condition nécessaire à toute conception. Cette parenté qui, enfin, semble en toutes circonstances s'articuler de façon privilégiée autour de la nomenclature. On relèvera au passage une nouvelle désinvolture de l'auteur de La parenté. Dans un ouvrage qui compte plus de huit cents pages, Barry n'en consacre que quatre à la question de la nomenclature, lui déniant ipso facto la moindre pertinence. Il regrette même que, depuis Rivers, le lien nécessaire entre filiation et mariage d'une part, nomenclature de l'autre, demeure pour les anthropologues le fondement de toute analyse de la parenté, explicitement ou implicitement (pp. 203, 71-75) ${ }^{16}$. Le procédé nous semble léger, qui consiste à congédier l'une des préoccupations constantes des spécialistes de la parenté. Il devient parfaitement irrecevable dans un contexte américaniste où, ainsi que Morgan l'avait pour le coup parfaitement compris dans un premier temps, la nomenclature fait la parenté, en l'absence le plus souvent, de règles de mariage prescriptives, et où la ligne de partage entre consanguinité et affinité ne revêt pas nécessairement la dichotomie radicale qu'elle possède en Occident car celleci n'est qu'un opérateur logique parmi d'autres servant à définir les positions et donc les appellations ${ }^{17}$. À cet égard, on pourrait rapprocher et opposer à la fois Barry et Kroeber : tous deux prétendent à l'universalité de leur leçon alors qu'ils raisonnent pareillement à partir de leur "base arrière ethnographique ", laquelle est partielle, constituée par le monde occidental et ledit «mariage arabe » d'un côté, par la seule Amérique du Nord de l'autre. Cela dit, les intuitions de Kroeber sur les déterminants « cognitifs »- si l'on peut se permettre un tel anachronisme épistémologique - de la nomenclature conservent jusqu'à nos jours toute leur profondeur ${ }^{18}$. On peut, dès lors, lui pardonner le biais américaniste de son inspiration, tout en profitant pleinement de sa leçon dans ce contexte précisément. En revanche, presque un siècle plus tard, il nous semble difficile, sauf à se complaire dans un idéalisme mondain, de s'affranchir de toute réflexion quant à la détermination culturelle éventuelle des faits ethnographiques produits à l'appui d'une quelconque théorie anthropologique générale et universelle. En bref, pour en revenir à la parenté amérindienne, il nous faut plus sûrement l'élucider à partir du Lévi-Strauss des Mythologiques qu'à travers celui-ci des Structures élémentaires de la parenté.

\section{NOTES}

1. «Il désire rendre visite ici à d'anciennes connaissances, amis ou parents.

- Il a de la parenté par ici?

- Oui et non, cela dépend comment on veut voir les choses ». 
2. Laurent Barry, La parenté, Gallimard, coll. « Folio », Paris, 2008.

3. En revanche, l'ouvrage est dépourvu d'index général, ce qui en fait un instrument un peu difficile à manier pour le chercheur.

4. Laurent Barry, La parenté recomposée. Figures peul de l'alliance sur les hauts plateaux de l'Adamaoua (Nord Cameroun), thèse de doctorat, Université de Paris X, Nanterre, 1996.

5. Lewis Henry Morgan, Systems of consanguinity and affinity of the human family, Smithsonian Institution, Smithsonian Contributions to Knowledge vol. 17, Washington, 1871.

6. David Schneider, American kinship. A cultural account, The University of Chicago Press, Chicago, 1968.

7. Eduardo Viveiros de Castro, "Alguns aspectos da afinidade no dravidianato amazônico ", in Eduardo Viveiros de Castro et Manuela Carneiro da Cunha (éds), Amazônia : etnologia e história indígena, Núcleo de Historia Indígena e do Indigenismo da Universidade de Sâo Paulo/Fundaçâo de Amparo à Pesquisa do Estado de Sâo Paulo, Sâo Paulo, 1993, pp. 149-210.

8. Jack Goody (éd.), L'évolution de la famille et du mariage en Europe, Paris, Armand Colin, 1985 [édition originale en anglais : 1983].

9. Jérôme Wilgaux, Le "mariage dans un degré rapproché ». Approche historique du mariage athénien à l'époque classique, thèse de doctorat, Université de Bordeaux, Bordeaux, 2000.

10. Françoise Héritier, L'exercice de la parenté, École des Hautes Études en sciences sociales / Gallimard/ Le Seuil, Paris, 1981: «nul doute que la nécessité de l'échange entre les groupes humains est particulièrement bien adaptée au traitement différent des germains de même sexe et de sexe opposé, et subséquemment des cousins parallèles et des cousins croisés, sans en être cependant la cause ou la raison d'être... » (p. 47). Nous remercions un des lecteurs anonymes du Journal pour avoir attiré notre attention sur ce passage.

11. Françoise Héritier, Les deux sœeurs et leur mère. Anthropologie de l'inceste, Odile Jacob, Paris, 1994. Selon Héritier, rappelons-le, deux sœurs, ou une mère et une fille, ne peuvent pas partager le lit d'un même homme, car elles mettraient, par son truchement, en contact leurs propres humeurs corporelles, lesquelles sont déjà identiques et donc incompatibles. La théorie a valeur universelle, évidemment. Or il se trouve que la polygamie sororale est omniprésente en Amérique du Nord et que l'on y rencontre, plus rarement il est vrai, des cas de mariage avec la fille et la mère en même temps. Le fait n'a pas totalement échappé à Héritier. La façon dont elle en rend compte manque toutefois singulièrement de rigueur: "Nous avons vu plus haut que la prohibition de l'inceste du deuxième type [...] n'est pas universelle. C'est un type d'union qui peut même être recherché. La raison en est que tout dépend de la manière dont doivent être appariées ou contrariées des choses identiques ou différentes dans telle ou telle circonstance. Le raisonnement de base est toujours le même : il est dangereux d'accomplir l'un ou l'autre de ces deux actes. Les règles sociales viennent donc en accompagnement de ces inquiétudes, préconisant ou empêchant l'union entre des personnes qui partagent, à des degrés divers, une identité substantielle " (pp. 246-247). En bref, l'interdiction de l'inceste du second type est en vigueur sauf là où il est pratiqué. Sa valeur heuristique n'en serait pas moins centrale. Malheureusement, à notre connaissance, les mariages sororaux nord-américains n'ont absolument rien de spéciaux aux yeux de ceux qui s'y adonnent... en ce sens, rien, dans l'ethnographie, ne permet d'avancer qu'ils possèdent un quelconque caractère incestueux.

12. À l'exception de la " dispute » entre Viveiros de Castro et Héritier au début des années 1990 sur l'éventuelle patrilinéarité sous-jacente des systèmes semi-complexes (voir Eduardo Viveiros de Castro, "Structures, régimes, stratégies ", L'Homme, 33 (125), 1993, pp. 117-137 ; Elisabeth Rougier-Copet et Françoise Héritier-Augé, "Commentaires sur commentaires, réponses à E. Viveiros de Castro ", L'Homme, 33 (125), 1993, pp. 139-148). Remarquons que les systèmes semi-complexes, chers à Lévi-Strauss et dont la bonne compréhension apparaissait si cruciale à une époque, ainsi que l'atteste cette dispute, semblent avoir sombré corps et biens depuis, du moins comme objet de discussions théoriques de premier rang.

Journal de la Société des américanistes, 95-1 | 2009 
13. Emmanuel Désveaux, «La consanguinité. Horizon indépassable de la raison parentaire ?", L'Homme, 164, 2002, pp. 105-124.

14. Anne Christine Taylor, "Jivaro kinship: "simple" and "complex" formulas: a dravidian transformation group ", in Maurice Godelier, Thomas Trautmann et Franklin E. Tjon Sie Fat (éds), Transformations of kinship, Smithsonian Institution Press, Washington, 1998; Eduardo Viveiros de Castro et Carlos Fausto, «La puissance et l'acte : la parenté dans les basses terres d'Amérique du Sud », L'Homme, 33 (126-128), 1993, pp. 141-170; Aparecida Vilaça, « Making kin out of others in Amazonia ", The Journal of the Royal Anthropological Institute, 8 (2), 2002, pp. 347-365; Isabelle Daillant, Sens dessus dessous. Organisation sociale et spatiale des Chimane d'Amazonie bolivienne, Société d'ethnologie, Nanterre, 2003. Voir également le volume collectif d'Eduardo Viveiros de Castro et Aparecida Vilaça (éds), Antropologia do parentesco: estudos ameríndios, Editora UFRJ, Rio de Janeiro, 1995.

15. Eduardo Viveiros de Castro, From the enemy's point of view. Humanity and divinity in an Amazonian society, The University of Chicago Press, Chicago, 1992.

16. Barry en profite au passage pour critiquer sévèrement les spécialistes des «basses terres d'Amazonie " à propos des incohérences que susciteraient dans leurs écrits les adéquations admises comme évidentes entre classes terminologiques et règles de mariage. Il attaque ici de façon anonyme. Nous laissons à nos différents collègues amazonistes le soin de se reporter au texte, très étonnant par la vigueur de sa charge, afin de savoir s'ils se sentent visés ou non...

17. Emmanuel Désveaux, Quadratura Americana. Essai d'anthropologie lévi-straussienne, Georg, Genève, 2001, chapitre XXI.

18. Alfred L. Kroeber, "Classificatory systems of relationship ", Journal of the Royal Anthropological Institute, 39, 1909, pp. 77-84.

INDEX

Thèmes : Anthropologie sociale

Mots-clés : parenté, Barry (Laurent)

Keywords : kinship, Barry (Laurent)

Palabras claves : parentesco, Barry (Laurent)

\section{AUTEUR}

\section{EMMANUEL DÉSVEAUX}

EHESS, 54, bd Raspail, 75006 Paris/Humboldt Universität, Institut für Europäische Ethnologie, Mohrenstrasse 41, 10117 Berlin. 